cessive PFC DA levels may be one of the mechanisms by which the Met genotype in 22q11DS is disrupting SR.

\section{Poster \#107 \\ GENETIC VARIATION UNDERLYING PSYCHOSIS-INDUCING EFFECTS OF CANNABIS: CRITICAL REVIEW AND FUTURE DIRECTIONS}

Jeroen Decoster ${ }^{1,2}$, Jim van Os ${ }^{1,3}$, Inez Myin-Germeys ${ }^{1}$, Marc De Hert ${ }^{2}$, Ruud van Winkel ${ }^{1,2}$

${ }^{1}$ Dept. of Psychiatry and Psychology, School for Mental Health and Neuroscience, EURON, Maastricht, Netherlands; ${ }^{2}$ University Psychiatric Centre Catholic University Louvain, Kortenberg, Belgium; ${ }^{3}$ King's College London, King's Health Partners, Department of Psychosis Studies, Institute of Psychiatry, London, United Kingdom

Background: Cannabis use is associated with an increased risk for psychotic disorder, yet most cannabis users do not develop psychosis. Therefore, any causal effect of cannabis may be rather modest and is likely explained by the fact that cannabis may only increase risk for psychosis in interaction with other environmental and genetic risk factors.

Methods: A systematic review of (i) the literature examining possible differential sensitivity to the psychotogenic effects of cannabis according to underlying genetic liability and (ii) the reported molecular genetic findings with regard to gene $\times$ cannabis in psychosis.

Results: There is robust evidence that persons at psychometric risk for psychosis are most vulnerable to display psychotic symptoms subsequent to the use of cannabis. Multiple studies have also found that persons at familial risk for psychosis have an increased sensitivity to the effects of cannabis. Together, these findings support the concept of a biological interaction between cannabis use and one's underlying genetic vulnerability, in terms of psychotic symptoms. Less evidence is available regarding possible underlying neurobiological changes, although the available studies consistently support differential sensitivity to cannabis use in terms of neurobiology as well. For cognition, the reported findings are inconsistent. At the molecular-genetic level, however, few (if any) interactions have been consistently replicated, although a reported interaction with variation in AKT1 is promising and deserves further follow-up. The apparent lack of consistent replication can be ascribed to problems of initial gene selection, statistical power, a bias towards positive results and insufficient attempts at true replication.

Discussion: In further research of gene-cannabis interaction in psychosis, increased sample sizes, greater density of genetic markers and a stronger focus on true replication are necessary. The major challenge for moleculargenetic gene-environment interaction research will be to combine the agnostic detection of disorder-associated genetic variants from genomewide studies with the hypothesis-based approach from epidemiological and neurobiological studies. Possible strategies for future cannabis interaction studies will be discussed.

\section{Poster \#108 \\ MODULATORY EFFECT OF THE INTERLEUKIN -1B GENE ON DORSOLATERAL PREFRONTAL CORTEX FUNCTION IN SCHIZOPHRENIA BUT NOT IN HEALTHY SUBJECTS: A GENETIC NEUROIMAGING STUDY}

Mar Fatió-Vilas ${ }^{1,2}$, Edith Pomarol-Clotet ${ }^{2,3}$, Raymond Salvador ${ }^{2,3}$, Gemma C. Monté ${ }^{2,3}$, Jesús J. Gomar ${ }^{2,3}$, Salvador Sarró ${ }^{2,3}$, Jordi Ortiz-Gill ${ }^{2,4,5}$, Candibel Aguirre ${ }^{3,4}$, Ramón Landín-Romero ${ }^{2,3}$, Amalia Guerrero-Pedraza ${ }^{3,4}$, Sergi Papiol ${ }^{1,2,6}$, Josep Blanch ${ }^{7}$, Peter J. McKenna ${ }^{2,3,4}$, Lourdes Fañanás ${ }^{1,2}$ ${ }^{1}$ Unitat d'Antropologia, Departament de Biologia Animal, Facultat de Biologia, Universitat de Barcelona. Institut de Biomedicina de la Universitat de Barcelona (IBUB), Barcelona, Spain; ${ }^{2}$ Centro de Investigación Biomédica en Red de Salud Mental (CIBERSAM), Spain; ${ }^{3}$ FIDMAG, Germanes Hospitalàries, Spain; ${ }^{4}$ Benito Menni Complex Assistencial en Salut Mental, Sant Boi de Llobregat, Spain; ${ }^{5}$ Hospital General de Granollers, Granollers, Spain; ${ }^{6}$ Max Planck Institute of Experimental Medicine, Clinical Neuroscience Division, Göttingen, Germany; ${ }^{7}$ Fundació Sant Joan de Déu, Barcelona, Spain

Background: Beyond their traditional role in the immune system, interleukins have been found to be involved in a variety of neural processes such as regulation of neuronal excitability, synaptic transmission, maturation and plasticity, glial migration and differentiation and other processes of brain development (Nawa et al 2006). Recent meta-analyses have confirmed that the interleukin-1 beta gene (IL1B, 2q13) influences the risk for schizophrenia (Allen et al 2008, Xu et al 2010), but the underlying biological mechanisms of this association are still unclear. Investigation of the effects of genetic variability in this gene on brain function could provide more information about its role in the disorder.

Methods: A biallelic base-exchange polymorphism at the promoter region of IL1B $(-511 C / T$, rs16944) was genotyped in 48 schizophrenia patients (DSM-IV-TR) and 46 age and gender matched controls. All participants underwent fMRI while performing the n-back task. The present study examined the effects of the $-511 \mathrm{C} / \mathrm{T}$ polymorphism on brain correlates of working memory performance in both schizophrenia and control subjects.

Results: The patients with schizophrenia showed expected differences from controls in brain activation during performance of a working memory task. In the sample as a whole, genetic variability at IL $1 B$ was associated with differential brain activation in a bilateral frontal region including the dorsolateral prefrontal cortex (DLPFC), suggesting an involvement of this gene in activation of this brain area.There was also a significant diagnosis $x$ genotype interaction effect in an overlapping frontal region: the IL1B polymorphism did not affect activation in the controls in this area, but the schizophrenia patients who were $\mathrm{T}$ carriers showed significantly higher activation than the $\mathrm{CC}$ homozygotes.

Discussion: The present findings suggest the involvement of the IL1B gene in the regulation of the DLPFC activation during performance of the nback working memory task. It also provides evidence of a disease-specific effect, in that the genetic modulatory effect is limited to patients with schizophrenia. This may in turn have potential to account for an apparent protective effect of the $T$ allele against schizophrenia found in two recent meta-analyses. Acknowledgements: Funding for this study was provided by: i) Instituto de Salud Carlos III (FISPI10/01071; CP07/00048; CP10/00596; 10/231); ii) Centro de Investigación en Red de Salud Mental (CIBERSAM); Intramural Project CIBERSAM (P03/2010)); iii) Ministerio de Educación y Ciencia (SAF2008-05674-C03-00); iv) Comissionat per a Universitats i Recerca del DIUE (2009SGR827, 2009SGR211).

\section{Poster \#109 \\ NEURITIN-1 GENE IS ASSOCIATED WITH RISK FOR SCHIZOPHRENIA-SPECTRUM DISORDERS AND THEIR AGE AT ONSET}

Mar Fatjó-Vilas ${ }^{1,2}$, Salvador Miret ${ }^{3}$, Mjosé Muñoz $^{4}$, Bárbara Arias ${ }^{1,2}$, Manuel Ignacio Ibáñez ${ }^{5}$, Luisa Lázaro ${ }^{6,2}$, Victor Peralta ${ }^{7}$, Manuel J. Cuesta ${ }^{7}$, Generós Ortet ${ }^{5}$, Lourdes Fañanás ${ }^{1,2}$

${ }^{1}$ Unitat d'Antropologia, Departament de Biologia Animal, Facultat de Biologia, Universitat de Barcelona. Institut de Biomedicina de la Universitat de Barcelona (IBUB), Barcelona, Spain; ${ }^{2}$ Centro de Investigación Biomédica en Red de Salud Mental (CIBERSAM), Spain; ${ }^{3}$ Centre de Salut Mental de Lleida, Servei de Salut Mental i Addiccions, Hospital Santa Maria, Lleida, Spain; ${ }^{4}$ Àrea d'Adolescents. Complex Assistencial en Salut Mental, Benito Menni, Sant Boi de Llobregat, Spain; ${ }^{5}$ Departament de Psicologia Bàsica, Clínica i Psicobiologia, Facultat de Ciències Humanes i Socials, Universitat Jaume I, Castelló, Spain; ${ }^{6}$ Servei de Psiquiatria i Psicologia Infantil i Juvenil, Hospital Clínic de Barcelona, Barcelona, Spain; ${ }^{7}$ Servicio de Psiquiatría, Hospital Virgen Camino, Pamplona, Spain

Background: Among the numerous features used to characterize psychotic disorders, age at onset of the disorder is widely accepted as having particularly strong etiological significance. The more severe clinical and cognitive profile associated to early onset forms has lead to the definition of these cases as a group with more salient genetic factors and, therefore, as a group of high interest to detect genetic factors associated with the disorder. Interestingly, the chromosomal region 6p22-p25 was identified as linked to a subtype of schizophrenia characterized by pervasive cognitive deficits (Hallmayer et al 2005). This region includes two genes involved in neurodevelopment and synaptic plasticity: dysbindin-1 gene (DTNBP1) and neuritin-1 gene (NRN1). DTNBP1 has been associated with schizophrenia and, more recently, also with cognitive deficits in early-onset psychotic disorders (Wessman et al 2009, Fatjó-Vilas et al 2011). However, the implication of NRN1 in these phenotypes is still far unknown. The aim of the present work was to investigate the association of NRN1 with: i) the risk for developing a psychotic disorder, ii) age at onset and cognitive functioning. 